\title{
Denoising Small Signals using Averaging Methods in Arduino
}

\author{
Z. Zaid ${ }^{1}$, I. Mustaffa ${ }^{2}$, M. M. M. Aminuddin', M. D. H. Gamal ${ }^{3}$ \\ ${ }^{1}$ Department of Electronic and Computer Engineering Technology, \\ Universiti Teknikal Malaysia Melaka, Hang Tuah Jaya, 76100 Durian Tunggal, Melaka, MALAYSIA \\ ${ }^{2}$ Department of Industrial Electronics, \\ Universiti Teknikal Malaysia Melaka, Hang Tuah Jaya, 76100 Durian Tunggal, Melaka, MALAYSIA \\ ${ }^{3}$ Department of Mathematics, \\ Universitas Riau, Pekanbaru, Riau, INDONESIA \\ *Corresponding Author
}

DOI: https://doi.org/10.30880/ijie.2019.11.04.034

Received 8 August 2019; Accepted 26 August 2019; Available online 5 September 2019

\begin{abstract}
Electroencephalograms (EEG) acquired from the scalp is a small amplitude and low-frequency signal, with noise accumulation which originates from spontaneous activity of the human brain. Averaging methods are frequently used in reducing noise in EEG signals during the post-processing stage and never in real time. The methods are frequently engaged in waveform measurements in order to reduce the additive noise and at the same time, retaining significant parts of the noisy instances. This paper describes averaging methods used in real-time. The processes are emulated using MATLAB and then implemented in Arduino. The correlation and the SNR between the input signal and output of the averaging methods were calculated and compared.
\end{abstract}

Keywords: Arduino; MATLAB; averaging; real-time acquisition

\section{Introduction}

An acquired biosignal is usually comprised of the desired signal along with additive noise. Electroencephalography (EEG) is a medical imaging technique that acquires the electrical activity of the brain from the scalp [2]. The neurons in the human brain are ceaselessly active, emitting some small amounts of electromagnetic waves signal, which only can be detected in the range of $0.03 \mathrm{~Hz}-50 \mathrm{~Hz}$ of frequency band [3] on certain wavebands, are used as EEG signals. When the signal comprises with too many undesirable signals, there may be the significantly small amount of application that can evaluate the signal appropriately.

Filtering can be achieved by many methods, and such is being used in this study is a method called moving average (MA). In statistics, MA is a calculation to analyze data points by forming a series of the average of different subsets of the full data set. As in electronics, MA is a simple low pass FIR (Finite Impulse Response) filter commonly used for regulating an array of sampled data or signal. There are various types of MA used in many different types of applications, such as Simple Moving Average, Weighted Moving Average and Exponential Moving Average.

Averaging is the most used method in denoising EEG signals to remove noise due to spontaneous activities despite the fact that there are all sorts of available digital signal filters. The definition of the acquired EEG signal being a summation of the EEG signal and the spontaneous activities of the brain contributes to the still widely used method of denoising EEG. The process normally takes place at the post-processing stage offline [4] since averaging requires a set of initial data before the method can be carried out. Here it is described of how the averaging technique is implemented in the real-time acquisition of the EEG signal.

In the following sections, the moving average system, the development of hardware and software are described. The results of the moving average and averaging methods using MATLAB and Arduino are shown and analyzed. 


\section{Moving Average and Averaging}

\subsection{Moving Average}

Moving average (MA) is used to reveal data trends associated with time which involves data smoothing. Hence, MA is also a filter as it removes low-frequency drift that happens in the input data or signal. The low-frequency drift is effectively removed by subtracting the output of the MA filter from the original filter [4] [5]. Simple Moving Average (SMA) is a common average of the previous $n$ data points in time series data. Each point in the time series data has equal weights, so there are no weighting factors applied to any of the data points [6]:

where,

$$
S M A=\frac{P_{M}+P_{M-1}+\cdots+P_{M-(n-1)}}{n}
$$

$P_{M}=$ data point at $\mathrm{M}$

$M=$ discrete time

$n=$ number of data points used

When calculating successive values, a new value comes into the formula's sum and the earliest data dismissed. SMA is often used to observe important trends in repeated statistical surveys. It is recognized as a lowpass FIR filter. SMA is optimal for a common task such as reducing random noise [7].

Weighted Moving Average (WMA) is an improved form of SMA. WMA assigns a weighting factor to each value in the data series according to its sequence. The most recent data gets the greatest weight and each price value gets a smaller weight. The weighting factors are calculated from the sum of times used in time series data, also known as the 'sum of digits'.

$$
W M A=\frac{n P_{M}+(n-1) P_{M-1}+\cdots+2 P_{(M-n+2)}+P_{(M-n+1)}}{n+(n-1)+\cdots+2+1}
$$

Exponential Moving Average (EMA) is the most recent kind of WMA which allocates weighting element to each value in the data series according to its time. EMA is proposed for detecting an increase in the mean of a sequence of random variables [8]. The difference between WMA and EMA is that in EMA the weighting for each previous data point decreases exponentially so that the point will never reach zero.

The EMA for a time series Y compute recursively [9]:

$$
\begin{gathered}
S_{1}=Y_{1}, \\
\text { for } t>1, S_{t}=a \cdot Y_{t}+(1-a) \cdot S_{t-1},
\end{gathered}
$$

where $Y_{t}$ is the value at time period $t, S_{t}$ is the value of EMA at time period $t$, and $\alpha$ represents the degree of weighting decrease, as a constant smoothing factor between the range of 0 until 1. Commonly, $\alpha$ is calculated using the formula:

$$
a=2 /(n+1)
$$

In different studies made by Braun [10], stated out that EMA is a recursive autoregressive (AR) form of expression known by:

$$
y(n)=y(n-1)+\frac{x(n])-y[n-1)}{x}
$$

where $K$ is a constant. A small value of $K$ has a reduced of a smoothing effect and is more reactive to recent changes in the data and vice versa. [10]. The more familiar expression is:

$$
y(n)=-a y(n-1)+(1-a) x(n)
$$

where $a=(1-1 / K)$,

It is relatively direct to show an equivalent MA expression:

$$
y(n)=\frac{1}{K}\left[e^{(00)} x(n)+e^{(q)} x(n-1)+e^{(2 q)}(n-2)+\cdots+e^{\left.(n-1) q_{x}(1)\right]}\right.
$$

where $q=\ln (1-1 / K)$ 
While the terms to the right behave like a decaying exponential, with less weight given to distant past data points. The focus of this paper is using the averaging method in real-time as a filter of the EEG signal.

\subsection{Averaging}

Averaging is a substantial method of denoising the noise of quasi-periodical or event-related signal [11]. Outliers such as spike artifacts or bursts of noise in the signal affect the analysis [12]. Averaging method turns out to come in handy, as it accomplishes as an ideal system with a group of delay of $(\mathrm{M}+1) / \mathrm{N}$ samples connect with both processing elements in a parallel form [13].

The traditional filtering schemes seem to be lacking when the signal and noise frequency spectra significantly overlap. Averaging, on the other hand, used for separating a repetitive signal from noise without introducing signal distortion. In [14], it was illustrated how averaging is an effective and simplest way to eliminate the harmonic thoroughly. As it is the one of the shortest method possible response time in some scenarios, the averaging application turns out to be another solution, particularly when selective cancellation is requiring for some specific disturbance components in the control loop. The average of a set of numerical values, compute by accumulate them together and divide it by the number of terms in the set, in order to find another value, known as the arithmetic mean. When arithmetic means is about to develop, where if $n$ numbers are given, each number represented by $a_{i}$ (where $I=1,2, \ldots$, $\mathrm{n})$, the arithmetic mean is the sum of the as divided by $n$ or:

$$
\mathrm{AM}=\frac{1}{n} \sum_{i=1}^{n} a_{i}=\frac{1}{n}\left(a_{1}+a_{2}+\cdots+a_{n}\right)
$$

The applications of analysis in biomedical signals, such as EEG and electrocardiograms (ECG) usually practice the averaging method [4] [15].

\subsection{Arduino}

Arduino is an open-source platform used for constructing and programming of electronics [16], where it sends and receives information and to control electronic devices. Arduino board are based on Atmel microcontroller units (MCU). Arduino Uno R3 board specifically with ATmega328P is widely used by many researchers due to its affordable cost. It has an easy programming language based on ready $\mathrm{C} / \mathrm{C}++$ libraries for serial communication, analog-to-digital (A/D) conversion, 2-wire interface protocols as well as many other embedded system application and is based on Atmel MCU's with AVR architecture [17] [18].

In signal processing, an FIR filter is a filter whose impulse response, or response to any finite length input, is of finite duration, due it settles to zero in finite time. MA is a special case of the regular FIR filter. MA method is implemented on the Arduino board where the algorithm of each MA method is being processed on the $\mathrm{C} / \mathrm{C}++$ language.

\section{Results and Discussion}

Data from the input signal of random noise (RAND) from the MATLAB are being used back in the Arduino as a replacement for the input signal. RAND stands for uniformly distributed random numbers, where it returns a single uniformly distributed random number in the range interval. As for this study, RAND10 is used as a replacement of input signal that supposed to be connected to the input of the Arduino. RAND10 means that it will return a random number in the range from 10 until -10. All of this value is a state in voltage (V), as it is implemented in the system that contains electrical voltage. As for the small signal, the amplitude is being converted into smaller value using a certain MATLAB command, where it changes the value into a small signal, which is in $\mu \mathrm{V}$.

Both SMA and WMA signal looked smooth enough in getting good, but at the same time, it also removed the important data on the signal as well. As for EMA, the shape of the signal was maintained, although the signal has been smoothed out. SMA showed less smooth up the signal than expected and some the frequency are not properly removed but removed some of the important data on it, and thus let through an amount of the signal shorter than the original. The same thing happens at the WMA, where it also smooth up the signal but removed some of the important data on it. The correlation shows that WMA has the lowest resolution of the correlation coefficient, followed by SMA and EMA has the highest resolution of it. Table 1 shows the result of how the signal-to-noise ratio while Table 2 shows the result of how the correlation coefficient for each for the signal using the different moving average method that has been tested out:

EMA illustrated a smoothed up the signal with less removing the data, due to the coefficient of $\alpha$ that represented the degree of weighting. Lag also happened in this method, but not too obvious, as it maintained the sharp-peak of the signal [20]. Fig. 1 shows how the comparison of each method towards the signal, as it is filtered in order to remove any unwanted signal by using MATLAB: 
Table 1 - Result for SNR for each of the signal using the SMA, WMA, and EMA that have been tested out

\begin{tabular}{cc}
\hline $\begin{array}{c}\text { Method of } \\
\text { MA }\end{array}$ & $\begin{array}{c}\text { Signal-to-Noise Ratio } \\
\text { (SNR) }\end{array}$ \\
\hline SMA & $2.5409 \mathrm{~dB}$ \\
WMA & $4.0586 \mathrm{~dB}$ \\
EMA & 7.5627 \\
\hline
\end{tabular}

Table 2 - Result for correlation coefficient for each of the signal using the SMA, WMA, and EMA that have been tested out

\begin{tabular}{cc}
\hline Method of MA & $\begin{array}{c}\text { Correlation } \\
\text { Coefficient }\end{array}$ \\
\hline SMA & 0.8244 \\
WMA & 0.7678 \\
EMA & 0.8737 \\
\hline
\end{tabular}

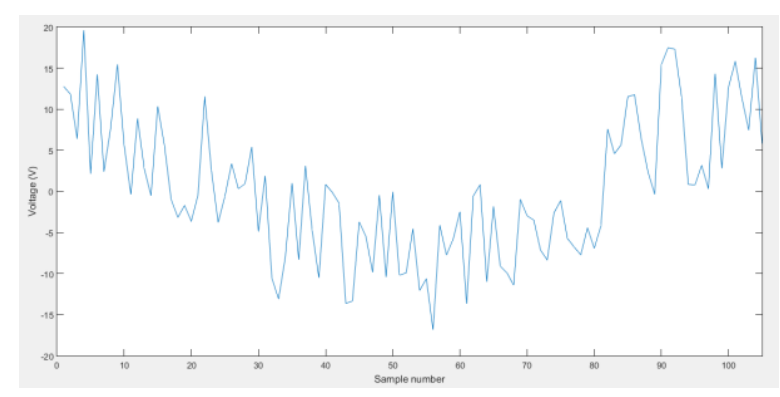

(a)

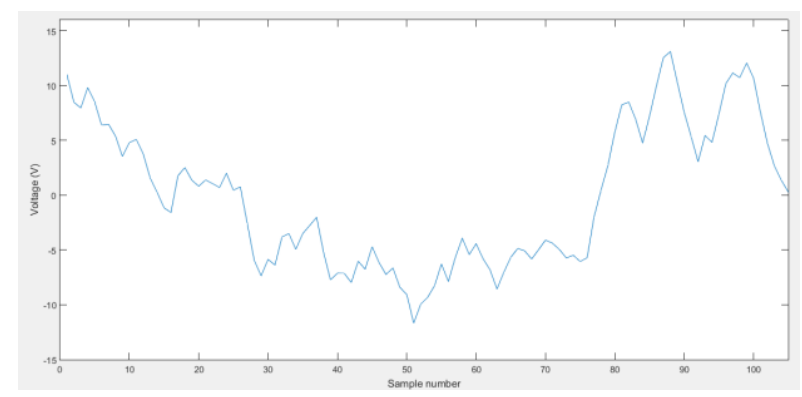

(c)

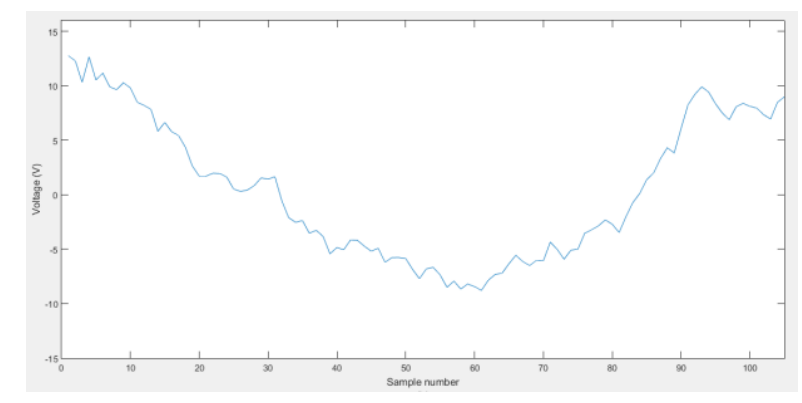

(b)

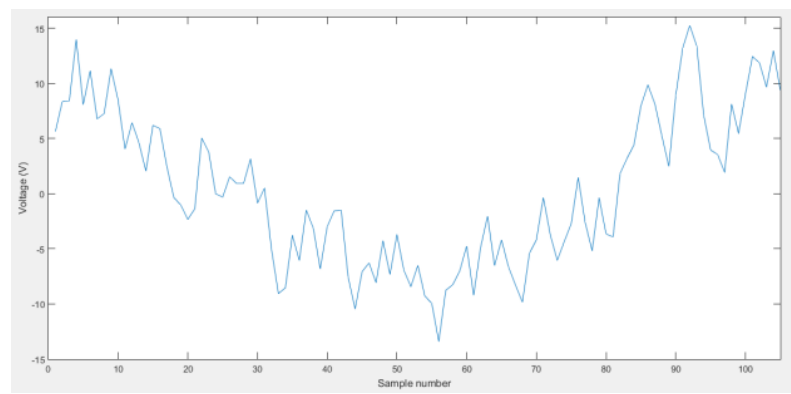

(d)

Fig. 1 - Comparison of moving average method towards the signal using MATLAB a) original input signal with noise b) SMA c) WMA d) EMA

As for Fig. 2 shows how the comparison of each method towards the signal, as it is filtered in order to remove any unwanted signal by using Arduino. The comparison of data of the MATLAB and Arduino are continued with applying small signal input to it. The small data is produced from the conversion of the unit from the MATLAB. The small signal input has the range of $1 \mu \mathrm{V}$ to $10 \mu \mathrm{V}$. The reason why applying the small signal input on the MATLAB and Arduino is to see whether there are major changes happens in both of the signal input. Fig. 3 shows how the small signal input being implemented in the MATLAB, and Fig. 4 shows the comparison of each data of SMA, WMA, and EMA that have implemented on the Arduino. 


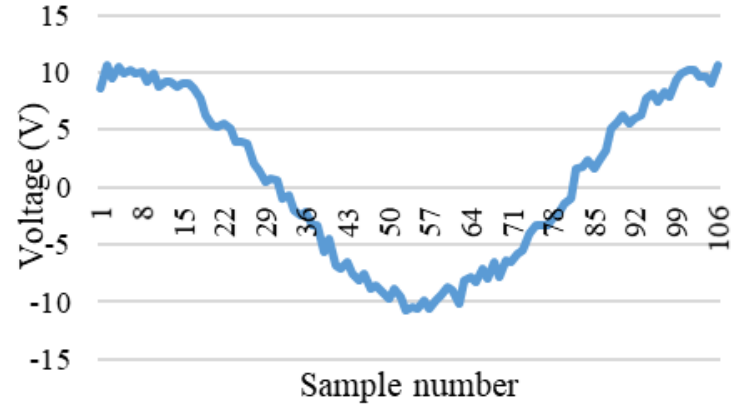

(a)

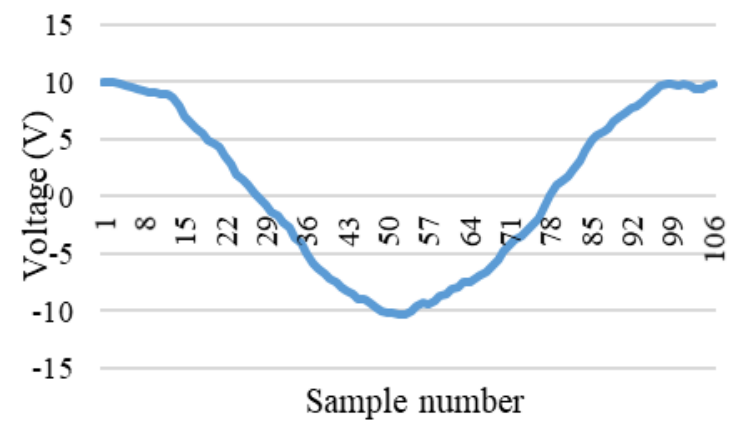

(c)

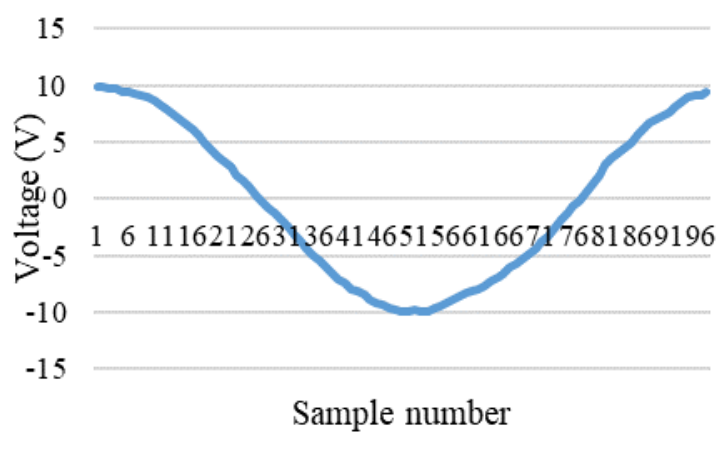

(b)

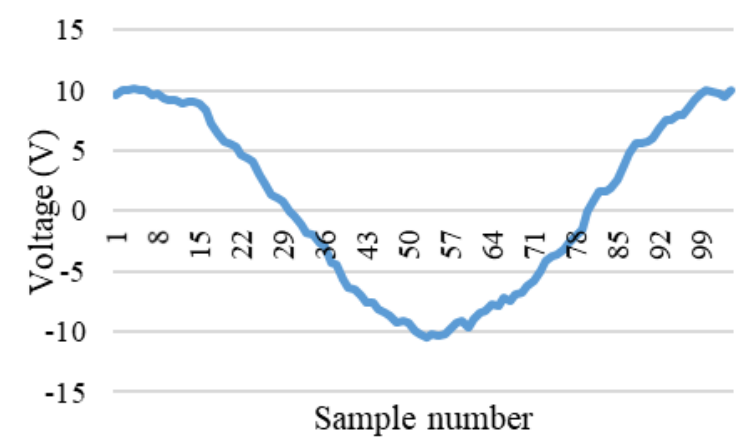

(d)

Fig. 2 - comparison of each moving average method towards the signal using Arduino a) original input signal with noise b) SMA c) WMA d) EMA.

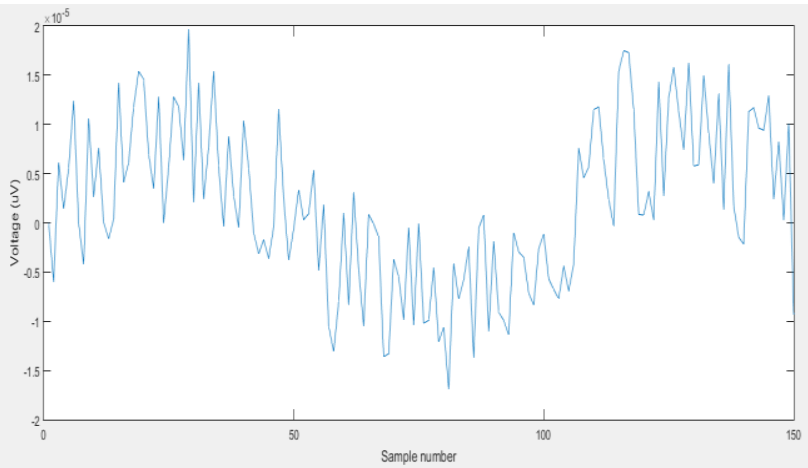

(a)

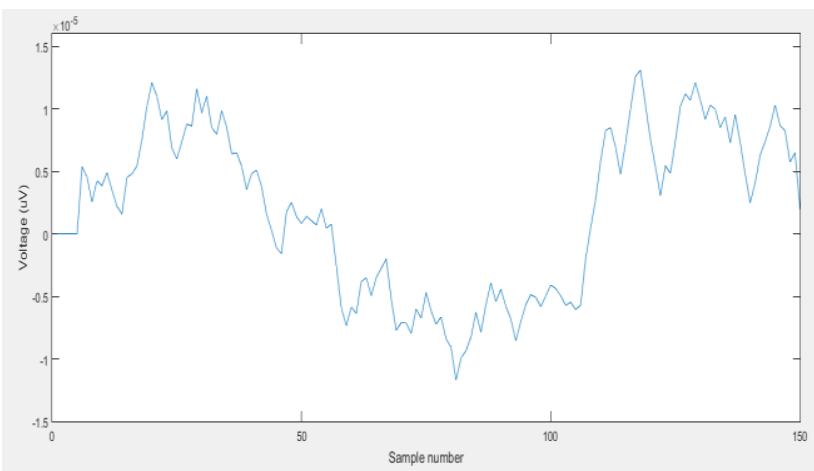

(c)

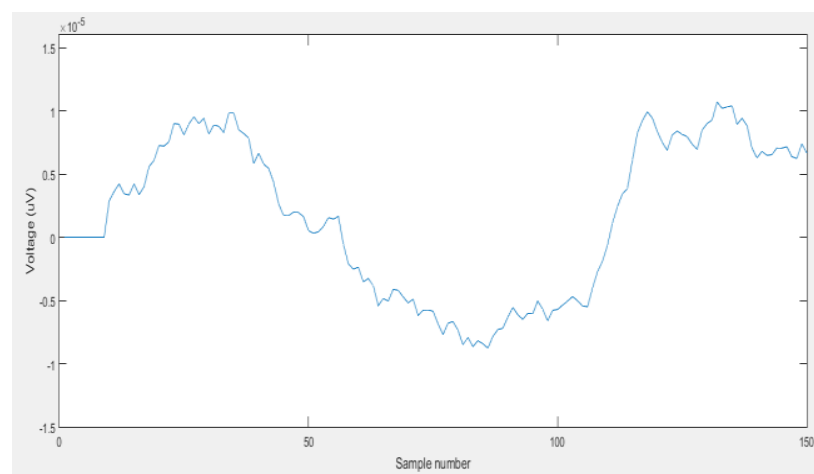

(b)

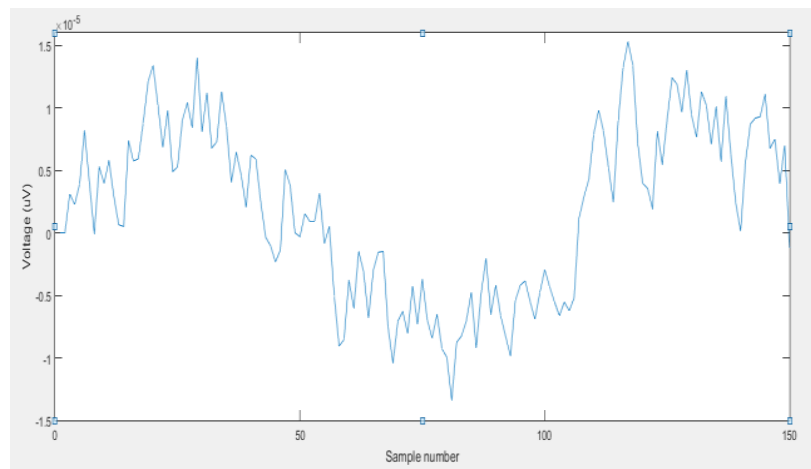

(d)

Fig. 3 - comparison of each moving average method towards the small signal using MATLAB a) original input signal with noise b) SMA c) WMA d) EMA. 


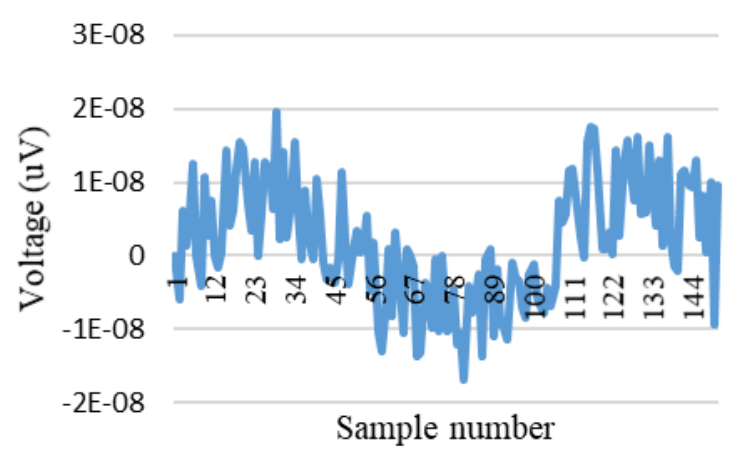

(a)

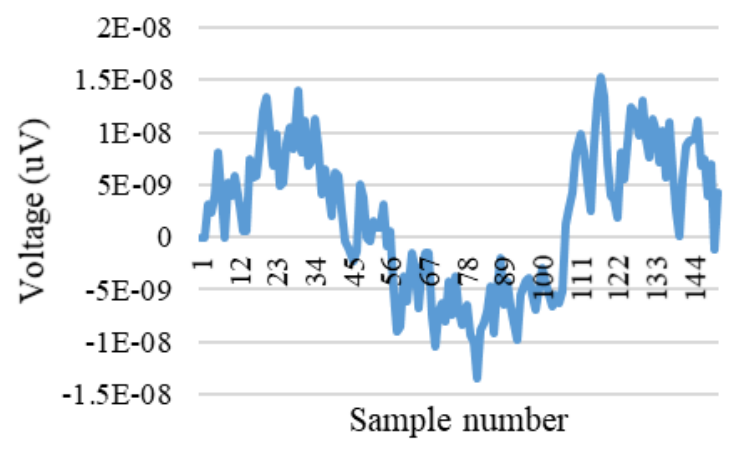

(c)

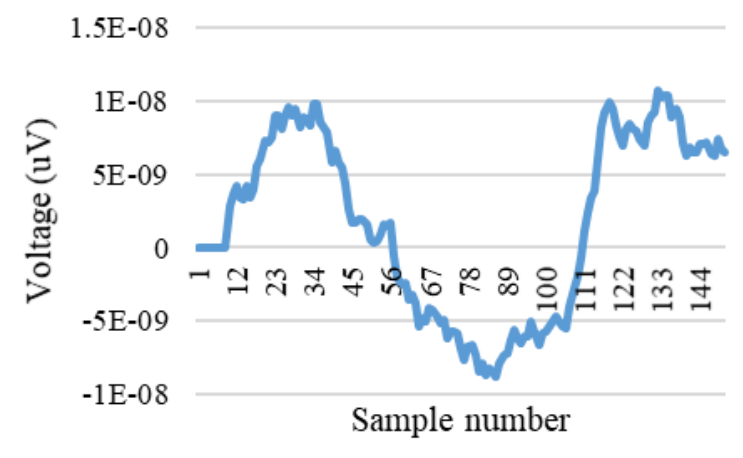

(b)

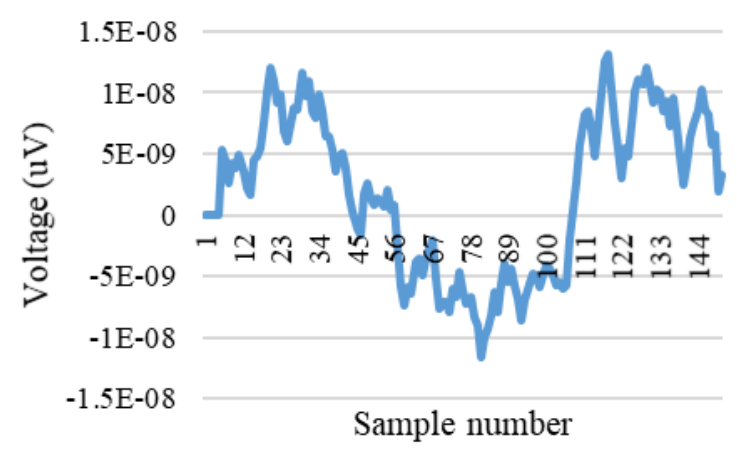

(d)

Fig. 4 - comparison of each method towards the small signal using Arduino a) original input signal with noise b) SMA c) WMA d) EMA.

\section{Summary}

EMA filtering was used and the best method compares to the SMA and WMA. EMA able to smooth up the signal that contains noise, as it does not remove important data on it as well. EMA also shows the most efficient quality signal after SNR test has been done in each of the MA methods, with EMA reach the highest value of 7.5627dB, compare to the SMA that have the lowest value of $2.5409 \mathrm{~dB}$, while WMA reaches the standard value of $4.0586 \mathrm{~dB}$ SNR. The correlation coefficient of the signal also proves that EMA has the highest efficiency, with 0.8757, compare to the other methods. A good filter must able to reach a certain specific range of SNR and correlation coefficient for the desired signal, in order to implement it in the system as the best method among the others. EMA proved to help in illustrating magnificent data and increasing the quality of SNR and efficiency of the desired signal into the specified level, with less removing the data, due to $\alpha$ that represents the degree of weighting.

\section{References}

[1] Motchenbacher, C. D., and Connelly, J. A. Low noise electronic design, Wiley-Interscience, Volume 19, No. 21, (1993).

[2] Teplan, M. Fundamentals of EEG measurement. Meas. Sci. Rev., Volume 2, No. 2, (2002), pp. 1-11.

[3] Liu, N. H., Chiang, C. Y., and Chu, H. C. Recognizing the degree of human attention using EEG signals from mobile sensors. Sensors (Switzerland), Volume 13, No. 8, (2013), pp. 10273-10286.

[4] Kotas, M., Pander, T., and Leski, J. M. Averaging of nonlinearly aligned signal cycles for noise suppression. Biomed. Signal Process. Control, Volume 21, (2015), pp. 157-168.

[5] Mustaffa, I., and Zaid, Z. Significance of averaging method signal denoising. Int. Conf. Robot. Autom. Sci., No. 1, (2017).

[6] Hansun, S. A new approach of moving average method in time series analysis," Univ. Multimed. Nusant., (2013).

[7] Azami, H., Mohammadi, K., and Bozorgtabar, B. An improved signal segmentation using moving average and Savitzky-Golay filter. J. Signal Inf. Process., Volume 3, No. 1, (2012), pp. 39-44.

[8] Ross, G. J., Adams, N. M., Tasoulis, D. K., and Hand, D. J. Exponentially weighted moving average charts for detecting concept drift. Pattern Recognit. Lett., Volume 33, No. 2, (2102), pp. 191-198.

[9] Proakis, J. G., and Monolakis, D. G. 4th Digital Signal Processing, 4th ed. USA: Pearson Education, 2007. 
[10] Braun, S. The synchronous (time domain) average revisited. Mech. Syst. Signal Process., Volume 25, No. 4, (2011), pp. 1087-1102.

[11] Kotas, M., Pander, T., and Leski, J. M. Averaging of nonlinearly aligned signal cycles for noise suppression. Biomed. Signal Process. Control, Volume 21, (2015), pp. 157-168.

[12] Pander, T. A new approach to robust, weighted signal averaging. Biocybern. Biomed. Eng., Volume 35, No. 4, (2015), pp. 317-327.

[13] Chen, H. C., and Chen, S. W. A moving average based filtering system with its application to real-time QRS detection. Comput. Cardiol., Volume 30, (2003), pp. 585-588.

[14] Xiong, L., Zhuo, F., Liu, X., Wang, F., and Chen, Y. Optimal design of moving average filter and its application in distorted grid synchronization," 2015 IEEE Energy Convers. Congr. Expo. 2015, (2015), pp. 3449-3454.

[15] Pandey, V., and Giri, V. K. High frequency noise removal from ECG using moving average filters," Int. Conf. Emerg. Trends Electr. Electron. Sustain. Energy Syst., (2016), pp. 1-5.

[16] Badamasi, Y. A. The working principle of an Arduino. Proc. 11th Int. Conf. Electron. Comput. Comput. ICECCO 2014, (2014).

[17] Cvjetkovic, V., and Matijevic, M. Overview of architectures with Arduino boards as building blocks for data acquisition and control systems. 2016 13th Int. Conf. Remote Eng. Virtual Instrum., No. February, (2016), pp. 5663.

[18] Busaidi, A. M. Development of an educational environment for online control of a biped robot using MATLAB and Arduino. 2012 13th Int. Work. Mechatronics, (2012), pp. 337-344.

[19] Filters, M. A. Moving average filters," Econ. Lett., Volume 37, No. 4, (1991), pp. 277-284.

[20] Ehlers, J. Signal analysis concepts. (2013), pp. 1-13. 